Mots. Les langages du politique

Le rapport, entre description et recommandation

\title{
« La vaccination, ça se discute? » Le rapport sur la politique vaccinale, espace polyphonique inédit
}

Is vaccination an issue for a public and contradictory debate? The parliamentary report about the vaccination policy, an unprecedented polyphonic text

La vacunación, ¿se discute? El informe de la politica vacunal, espacio polifónico inédito

Caroline Ollivier-Yaniv

\section{OpenEdition} Journals

Édition électronique

URL : https://journals.openedition.org/mots/22815

DOI : $10.4000 /$ mots. 22815

ISSN : 1960-6001

\section{Éditeur}

ENS Éditions

\section{Édition imprimée}

Date de publication : 10 juillet 2017

Pagination : 117-133

ISSN : 0243-6450

\section{Référence électronique}

Caroline Ollivier-Yaniv « «La vaccination, ça se discute? » Le rapport sur la politique vaccinale, espace polyphonique inédit », Mots. Les langages du politique [En ligne], 114 | 2017, mis en ligne le 10 juillet 2019, consulté le 22 avril 2022. URL : http://journals.openedition.org/mots/22815 ; DOI : https:// doi.org/10.4000/mots. 22815 


\section{"La vaccination, ça se discute? " Le rapport sur la politique vaccinale, espace polyphonique inédit}

Les vaccins et la vaccination sont devenus des objets politiques. Les débats qui les caractérisent et qui impliquent différents groupes d'acteurs dans des arènes publiques comme dans des espaces discrets et confinés (Gilbert et Henry, 2012) constituent des lieux d'observation privilégiés de l'état des relations entre les institutions, la science et la société.

Le 12 janvier 2016 a été rendu public un rapport commandé près d'un an plus tôt par le Premier ministre français à une députée socialiste : le « Rapport sur la politique vaccinale $»^{1}$ - encore appelé «Rapport Hurel» - a fait l'objet d'une conférence de presse de la ministre de la Santé et a suscité des retombées médiatiques immédiates dans les médias dits «de référence »². De prime abord, la commande et l'élaboration de ce rapport relèvent de pratiques routinières dans la conduite de l'action publique et en l'occurrence, du processus de transformation de la politique vaccinale. D'un strict point de vue chronologique, ce rapport apparaît de fait dans la continuité d'autres productions textuelles institutionnelles - avis ou rapports (France, 2012 et 2013) - dont la généalogie montre qu'ils relèvent du «programme national d'amélioration de la politique vaccinale 2012-2017»3.

Pour autant, ce rapport présente des particularités fortes si l'on considère à la fois sa teneur et son contexte politique et social d'élaboration. Tous les textes institutionnels récents portant sur la politique vaccinale, émanant des pouvoirs publics français comme de l'Organisation mondiale de la santé (OMS), commencent par faire le constat de l'insuffisance de la couverture vaccinale et d'une

1. Rédigé par Sandrine Hurel, députée de la Seine-Maritime jusqu'au 27 août 2015 et remis au Premier Ministre en janvier 2016, il sera désigné dans la suite du texte par le terme générique de «Rapport». Tous les passages soulignés le sont de notre fait.

2. Le soir même, le quotidien Le Monde consacre sa "Une » et son «Éditorial » à l'une des perspectives qui n'est pourtant qu'envisagée par le rapport, à savoir la fin de l'obligation en matière de vaccination.

3. Lettre de mission du Premier ministre à la députée Sandrine Hurel, Paris, 27 février 2015 (Hurel, 2016, p. 95-96). 
érosion de la confiance des populations envers les vaccins, en même temps qu'ils rappellent que la vaccination est l'action de santé publique la plus efficace qui soit. Tout en constituant une politique de santé publique paradigmatique et exemplaire, la politique vaccinale fait l'objet, en particulier en France, de controverses sur l'opportunité et les risques de transformer la loi rendant obligatoire la vaccination contre trois maladies (diphtérie, tétanos, poliomyélite) pour la population générale4, tandis que d'autres ne sont que recommandées. De fait, pour deux des principaux acteurs impliqués dans la réalisation de ce rapport5 puis dans la Concertation citoyenne sur la vaccination qui actualise l'une de ses recommandations ${ }^{6}$, l'objectif majeur de ce rapport est bien d'envisager une « rénovation de la politique vaccinale en France »7 et de mettre en discussion le devenir de l'obligation vaccinale, historiquement emblématique de la politique de santé publique française ${ }^{8}$.

Or le rapport Hurel se démarque nettement des avis et des rapports institutionnels qui l'ont précédé si l'on examine les acteurs qui ont été auditionnés : aux «personnalités qualifiées » habituellement sollicitées - et qui sont toutes des professionnels du monde médical (en immunologie, en pédiatrie, des représentants des ordres et des académies), de la recherche ou de la santé publique, le rapport Hurel ajoute des représentants de la société civile (associations de patients ou d'usagers) et des représentants de l'industrie pharmaceutique9. Enfin le contexte d'élaboration du rapport sur la politique vaccinale a été marqué par une importante controverse relative à une pétition initiée en ligne en mai 2015, dénonçant la pénurie des vaccins multivalents obligatoires (diphtérie, tétanos, poliomyélite) et réactivant les arguments relatifs à la dangerosité du vaccin contre l'hépatite $B$, aux risques liés aux adjuvants aluminiques et à l'emprise des laboratoires pharmaceutiques sur les pouvoirs publics ${ }^{10}$.

4. En France, seule la variole a fait l'objet d'une vaccination obligatoire. Instituée en 1902, elle a été abolie en 1984 .

5. Entretiens avec l'auteure, les 7 et 13 juillet 2016.

6. Site officiel de la Concertation citoyenne sur la vaccination : «http://concertation-vaccination. fr> (consulté le 5 avril 2017).

7. Pour reprendre le titre de l'intervention de la ministre de la Santé lors de la conférence de presse de présentation du rapport, le 12 janvier 2016.

8. D’abord parce que les usages répétés de la périphrase consistant à désigner la France comme «le pays de Pasteur » est significative du poids de l'histoire française de la vaccination; ensuite parce que la France est le dernier pays d’Europe à maintenir des obligations vaccinales en population générale.

9. La catégorie de l'expertise ne paraît pas la plus pertinente pour différencier les acteurs sociaux et ne sera donc pas particulièrement mobilisée. On retrouve en effet dans le monde du vaccin certaines des conclusions des travaux sur les associations de patients dans le contexte de démocratie sanitaire, qui ont mis en évidence l'importance de la notion d' "expertise profane», qu'elle soit fondée sur l'expérience et le témoignage ou sur l'appropriation de connaissances académiques et scientifiques par l'engagement associatif et plus généralement une socialisation secondaire (Akrich et Rabeharisoa, 2012).

10. Pétition en ligne intitulée «Vaccin obligatoire : les Français piégés par la loi et par les laboratoires!», initiée par Henri Joyeux, ancien cancérologue radié de l’Ordre national des médecins en juillet 2016, et par l'Institut pour la protection de la santé naturelle. 
Si les analyses socio-historiques de la vaccination s'accordent à souligner la récurrence des oppositions qui se sont manifestées à l'égard de son caractère obligatoire (Moulin, 1996), au point de considérer qu'elle peut être caractérisée par une «situation controversée durable » (Torny, 2011 ; Bertrand et Torny, 2004), il est nécessaire de bien différencier les débats relatifs à la politique vaccinale - en tant que politique publique -, qui se déploient dans les institutions et dont rendent compte les rapports ou avis qui ont précédé le Rapport Hurel, et les controverses relatives à certains vaccins et ou à leurs éventuels effets indésirables - qui se développent dans des arènes beaucoup plus ouvertes, médiatiques, et qui relèvent de mécanismes de construction des problèmes publics.

Dans la perspective d'une approche symbolique des problèmes publics (Céfaï, 2009; Gusfield, 2009), ces débats et ces controverses n’impliquent pas nécessairement les mêmes acteurs et surtout ne se déroulent pas dans les mêmes arènes. Comme l'a mis en évidence Joseph Gusfield en définissant un modèle constructiviste et symbolique des problèmes publics, les acteurs ou les groupes d'acteurs ont une capacité inégale à accéder aux différentes arènes de débat et certains peuvent être conçus comme des «propriétaires de problèmes publics» (Gusfield, 2009, p.10-11), même si cette situation est par définition changeante. Et comme l'ont par ailleurs souligné des travaux portant spécifiquement sur la définition des problèmes publics, au sens de la carrière d'un problème aboutissant à sa mise à l'agenda des pouvoirs publics (Hilgartner et Bosk, 1988 ; Gilbert et Henry, 2012) les arènes de débats peuvent être différenciées selon leur degré de publicisation ou au contraire, de discrétion et même de confinement. Or une revue des travaux sur les controverses vaccinales permet de dégager l'existence d'arènes bien différenciées, aussi bien du point de vue des lieux ou des dispositifs que des groupes d'acteurs qui les investissent : les réseaux sociaux et les forums de discussion configurent l'arène des critiques et des témoignages profanes, des acteurs sociaux non-institutionnels ou profanes-experts, cherchant pour certains à être reconnus comme lanceurs d'alertes (Ward et alii, 2015); les rapports et les commissions institutionnelles constituent une autre arène, confinée et investie par les experts authentifiés et les acteurs institutionnels; les médias classiques sont majoritairement investis par les acteurs les plus légitimes (qui sont les sources habituelles des journalistes), même s'il leur arrive de se faire le relais de controverses en ligne remarquables comme ce fut par exemple le cas pour la «pétition Joyeux» au printemps 2015 (Ollivier-Yaniv, 2016).

La production du rapport Hurel s'inscrit donc dans un contexte caractérisé à la fois par l'hétérogénéité - et probablement l'étanchéité - des arènes de débat plus ou moins publiques, comme des groupes d'acteurs qui s'y déploient. Dans cet article, on mettra en évidence que la teneur du texte du rapport est rendue remarquable par l'ampleur de sa dimension polyphonique, au sens où des 
voix à la fois nombreuses ${ }^{11}$ et très rarement rassemblées y sont repérables. On peut rapporter ce phénomène à l'audition d'acteurs sociaux exceptionnellement diversifiés et qui, pour certains d'entre eux, entretiennent des échanges agonistiques et des rapports de force en dehors de l'arène que figure ce rapport. Ces acteurs peuvent être considérés comme étant en situation d'antagonisme, même si, ou peut-être parce qu'ils ne font habituellement pas valoir leurs arguments dans les mêmes arènes, ni lors de débats directs. Considérant la matérialité discursive du rapport, celui-ci ne cite pas explicitement les «personnes rencontrées» (Hurel, 2016, p. 97) dans le cadre de son élaboration; il comporte en revanche des traces d'interdiscours significatifs de la reconnaissance de positions discordantes, clivées et clivantes, ou encore d'imputation de responsabilités antagonistes sur la politique vaccinale et, ou sur les vaccins.

La réflexion sera construite autour de deux hypothèses. Dans un premier temps, je montrerai que tout en assumant et en respectant sa tutelle par les pouvoirs publics et politiques de santé, le texte du rapport, tout comme les auditions et les sources bibliographiques mobilisées, assurent la reconnaissance de la pluralité des acteurs - qu'ils soient institutionnels ou non-institutionnels, dominants ou subalternes - qui entendent intervenir sur la redéfinition de la politique vaccinale, sans méconnaître leur mésentente, leur hostilité, voire l'incompatibilité de leurs positions. Autrement dit, ces acteurs apparaissent dans l'arène du rapport comme des «adversaires dont les idées peuvent être combattues [...] sans que jamais, cependant, leur droit à les défendre puisse être mis en question» (Mouffe, 2004, p. 186). On mettra ainsi en évidence que les acteurs porteurs de critiques relatives aux effets indésirables des vaccins, les associations de patients et d'usagers en particulier, se trouvent crédités d'une reconnaissance et d'une visibilité dans et par un texte institutionnel.

Dans un second temps, je soulignerai que les mésententes, et en l'occurrence les malentendus et les tensions, que permet de mettre au jour l'analyse du rapport ne sont pas réduites à un clivage entre acteurs institutionnels et acteurs scientifiques d'une part, et acteurs non institutionnels et profanes d'autre part. En soulignant certains débats internes aux institutions et aux professionnels du système de santé français, le rapport participe donc au déconfinement d'échanges communément restreints aux arcanes administratifs.

S'inscrivant dans la perspective d'une approche symbolique des problèmes publics ${ }^{12}$ (Gusfield, 2005), la présente analyse donne une centralité au rapport sur la politique vaccinale, en éclairant sa dimension interdiscursive par l'analyse de plusieurs corpus qui l'environnent et qui sont significatifs des acteurs impliqués dans les «luttes définitionnelles» (Gilbert et Henry, 2012) en cours

11. 127 personnes selon la «Liste des personnes rencontrées ( (Hurel, 2016, p. 97-102).

12. Au sens d'un « processus au travers duquel un état de fait devient un enjeu de réflexion et de protestation publiques et une ressource et une cible pour l'action publique» (Cefaï et Trom, 2005, p. 217). 
dans d'autres arènes : documents institutionnels (avis du Haut Conseil de la santé publique de mars 2014, rapports précédents); publications savantes ou semi-savantes sur l'évolution de la politique vaccinale et émanant d'experts (Bertrand et Torny, 2004; Vilde, 2015, blog de l'ancien directeur général de la santé William Dab, références bibliographiques figurant dans le rapport analysé) ; corpus d'articles de presse portant sur la pétition d'H. Joyeux en mai $2015^{13}$; enfin quatre entretiens ${ }^{14}$ avec des acteurs impliqués dans le phénomène étudié (production du rapport ou concertation citoyenne sur la vaccination) ${ }^{15}$.

\section{Le rapport, produit et instrument de la reconnaissance d'une pluralité d'acteurs intéressés par la redéfinition de la politique vaccinale}

Comme il est d'usage pour ce type de production textuelle institutionnelle, le rapport sur la politique vaccinale s'inscrit dans un processus caractéristique de l'action publique et, plus concrètement encore, du travail des acteurs publics et politiques. De fait, le texte du rapport comporte plusieurs mentions explicitant son inscription dans un processus institutionnel sectoriel et gouvernemental. Le rapport relève en effet d'une charge de mission auprès de la ministre de la santé qui émane du Premier ministre, notifiée par une lettre de mission en date du 27 février 2015, laquelle est insérée à la fin du rapport - après la liste des vingt recommandations. Cette lettre, comme le début du rapport, rappellent également son inscription dans un programme de travail ministériel, le «programme national d'amélioration de la politique vaccinale 2012-2017», dont il est signifié à plusieurs reprises dans le rapport qu'il est piloté par la Direction générale de la santé du ministère. Ce programme a déjà donné lieu à plusieurs travaux et à des documents (Conférence nationale de santé, 2012 ; Haut Conseil de la santé publique, 2014) produits par des acteurs administratifs de la Direction générale de la santé ou des membres des agences sanitaires, lesquels documents sont demeurés confinés aux arcanes institutionnelles.

Dans ce cadre institutionnel classique, la production du rapport repose sur l'élargissement des acteurs pris en considération, par rapport à ceux qui sont impliqués dans les échanges techniques, administratifs et spécialisés,

13. Corpus de 152 articles de la presse quotidienne nationale et de la presse quotidienne régionale publiés de mai à décembre 2015 constitué à partir de la base de données «Europresse».

14. Entretiens avec un président d'association de patients (4 juillet 2016), un professeur spécialiste de pathologie neuromusculaire (6 juillet 2016), un professeur d'immunologie pédiatrique (7 juillet 2016) et la députée chargée de mission pour l'élaboration du rapport (13 juillet 2016).

15. L'analyse des matériaux repose également sur l'interconnaissance professionnelle entre l'auteure et des professeurs en immunologie menant des recherches sur de nouveaux vaccins, du fait de son intégration dans un laboratoire biomédical ainsi que de sa participation aux enseignements du Master qui lui est adossé. 
mais aussi par rapport à ceux qui sont cités dans les médias classiques lors de controverses de fait caractérisées par la présence médiatique hégémonique des représentants des institutions publiques et des agences sanitaires, ainsi que des sociétés savantes et des organisations professionnelles médicales.

Plusieurs observations étayent cet élargissement dans et par le rapport Hurel. La liste des personnes auditionnées en premier lieu ${ }^{16}$ : celle-ci comprend les représentants de huit associations de patients et d'usagers aux positionnements et aux revendications diversifiés (prise en considération des effets indésirables de certains vaccins ${ }^{17}$ ou des adjuvants ${ }^{18}$, liberté d'information et de choix des individus en matière de vaccination ${ }^{19}$ ). Si le format du présent article ne permet pas de développer les arguments et les répertoires d'action de ces groupes, leur présence dans une production institutionnelle n'en reste pas moins inédite par rapport aux autres productions institutionnelles du même genre, comme en témoignent les commentaires qui justifient cette démarche, comme pour se défendre de transgresser la doxa institutionnelle. Par exemple, après avoir fait état de la position du Réseau vaccin hépatite $B$ relativement à la pénurie d'un produit vaccinal multivalent obligatoire ne contenant pas la valence hépatite $B$, le rapport précise la position de cette association en recourant à une formulation négative, faisant ainsi en sorte d'anticiper une objection qui pourrait être opposée à cet élargissement des acteurs auditionnés :

L'association REVAHB (Réseau vaccin hépatite B) regroupe les patients qui se considèrent victimes du vaccin contre l'hépatite $B$ [...] /l ne s'agit pas d'une ligue anti-vaccinale. (Hurel, 2016, p.76)

Même sans faire état des positions des différentes associations, le rapport rend compte d'une situation beaucoup plus complexe que la simple opposition à la vaccination en général, qui prend généralement la forme d’un antagonisme «anti-vax» (généralement emblématique des «ligues anti-vaccinales») versus "pro-vax». Il présente ce faisant une dimension prescriptive et transformante à l'égard de la position des institutions vis-à-vis du grand public, en incitant à l'écoute des interrogations et des doutes.

Améliorer l'adhésion des usagers et des professionnels de santé à la vaccination implique de redonner confiance dans la vaccination. Cela nécessite notamment de prendre en compte l'ensemble des doutes qui peuvent conduire à rejeter certaines

16. Liste dont la députée chargée de mission déclare qu'elle a été élaborée avec l'Inspectrice générale des affaires sociales qui l'accompagnait pour élaborer le rapport, « en toute indépendance » : «il n'y a pas eu d'arbitrage ailleurs, puisque à partir du moment où un parlementaire est en mission, il mène sa mission comme bon lui semble.» (Entretien avec l'auteure, 13 juillet 2016)

17. Collectif hépatites virales (CHV), Réseau vaccin hépatite B (REVAHB).

18. Association Entraide aux malades de myofasciites à macrophages (E3M).

19. Union nationale des associations citoyennes de santé; Association liberté, information, santé (ALIS), émanant de l'historique Ligne nationale pour la liberté des vaccinations, initialement Ligne nationale contre les vaccinations, créée en 1954 en opposition à l'obligation vaccinale (Bertrand et Torny, 2004). 
vaccinations et de répondre le plus précisément possible aux questions que se posent certains usagers et professionnels de santé. (Hurel, 2016, p.14)

Parallèlement est souligné le contraste entre l'importante utilisation des réseaux sociaux par les associations, ainsi que lors de mobilisations qui sont en l'occurrence évoquées de manière indirecte ${ }^{20}$, et l'absence des institutions sanitaires dans l'arène publique numérique.

Une communication officielle sur les raisons des ruptures de stock est indispensable afin de mieux expliquer la réalité de ces ruptures de stocks parfois présentées notamment sur les réseaux sociaux comme des stratégies délibérées des laboratoires pour augmenter leur profit.

Les informations diffusées par les réseaux sociaux constituent aujourd'hui un moyen de communication à ne pas négliger. L'INPES [Institut national de prévention et d'éducation à la santé] doit intervenir de façon structurée et officielle sur les réseaux sociaux. L'INPES puis la nouvelle agence nationale de santé publique devrait également apporter des informations en utilisant ce type de vecteur.

Recommandation no 3 : L'INPES doit intervenir de façon structurée et officielle sur les réseaux sociaux. (Hurel, 2016, p.49)

La thèse de l'inégalité d'accès des groupes d'acteurs aux arènes de débat (Gusfield, 2009) se trouve ainsi actualisée d'une manière en quelque sorte radicale : tout se passe comme si le rapport entérinait l'existence d'arènes publiques étanches entre elles et relativement peu concurrentielles. Les unes - les arènes numériques - sont investies par les associations aux positions hétérodoxes, tandis que les autres - les arènes médiatiques légitimes, ainsi que les arcanes institutionnelles sur lesquelles on reviendra dans la seconde partie - sont caractérisées par l'hégémonie des acteurs institutionnels sanitaires, médicaux et scientifiques.

Ce phénomène inédit d'élargissement des acteurs impliqués dans la démarche de redéfinition de la politique vaccinale passe également par l'audition d'un autre groupe peu visible dans les arènes publiques, pour des raisons bien différentes des associations : il s'agit des représentants de l'industrie pharmaceutique. En l'occurrence ont été entendus, en une seule audition ${ }^{21}$, cinq représentants de quatre grands groupes pharmaceutiques ainsi que deux représentants du syndicat des entreprises du médicament (LEEM). Le texte du rapport souligne que «lors des auditions, la question de la disponibilité des vaccins a été abordée à de nombreuses reprises» (Hurel, 2016, p. 91), mais encore que les laboratoires font globalement l'objet d'une suspicion²2.

20. Telle la pétition lancée en mai 2015 par H. Joyeux dénonçant notamment les ruptures de stocks des vaccins tétra- et pentavalents : «La mise en ligne d’une pétition mélangeant des fausses informations mais insistant sur la pénurie réelle de certains vaccins a contribué à ce climat de défiance» (Hurel, 2016, p. 76).

21. Entretien avec l'auteure, 13 juillet 2016.

22. «Impression pour certains d’une organisation de la pénurie (en vaccin pentavalent) par les 
La dénonciation de l'emprise des laboratoires sur les acteurs politiques et publics, mais encore de la domination des intérêts financiers et capitalistiques sur l'intérêt général et les enjeux de santé publique rassemble de fait des acteurs associatifs 23 et des élus de partis se situant à gauche de l'échiquier politique, ces derniers n'ayant pas été auditionnés 24 . Se trouve ainsi vérifiée l'hypothèse selon laquelle «les groupes se trouvant en affinité avec le jeu des oppositions partisanes bénéficieront de relais favorisant leurs interventions dans les arènes publiques » (Juhem et Sedel, 2016, p. 14).

Le rapport ne comporte aucune référence - pas plus implicite qu'explicite à ces dénonciations, celles-ci étant par ailleurs considérées par la députée responsable du rapport comme relevant de la théorie du complot 25 . Les «marges de manœuvre » dont disposent les laboratoires ainsi que «la difficulté à quantifier les marges prises par les laboratoires en complément de leurs coûts de fabrication » sont néanmoins soulignées (Hurel, 2016, p. 61). L'extrême complexité de l'anticipation et de la gestion des ruptures de stocks est également bien mise en évidence, notamment sur la base de comparaisons avec des pays dans lesquels les achats de vaccins sont centralisés. Le rapport n'en affirme pas moins clairement la nécessité, pour les institutions sanitaires, de reprendre la main sur les laboratoires sur au moins deux points. Le premier consiste à renforcer le pouvoir de la Haute autorité de santé (HAS) en matière de réalisation des évaluations médico-économiques préalables à la commercialisation des vaccins ainsi qu'en ce qui concerne l'inscription des événements indésirables dans le répertoire européen dédié26 - afin que ces tâches ne soient plus réalisées par les fabricants eux-mêmes. Le second point consiste à réaffirmer la nécessaire indépendance de l'organe consultatif national - pour la France, le Centre technique de la vaccination (CTV), rattaché au Haut Comité de santé publique - relativement aux fabricants de médicaments. Le rapport s'appuie en l'occurrence sur un article portant sur la gouvernance des politiques vaccinales, paru en 2010 dans la très légitime revue Vaccine, et en retire que les

laboratoires pour des raisons financières, malgré les informations délivrées par l'ANSM [Agence nationale de sécurité du médicament et des produits de santé]» (Hurel, 2016, p. 91).

23. Entretien avec un responsable d'association de patients, 4 juillet 2016.

24. Comme notamment la députée européenne Europe Écologie-Les Verts Michèle Rivasi, qui intervient régulièrement dans les médias, sur son blog et sur les réseaux sociaux pour dénoncer la primauté des intérêts financiers sur la santé publique tant en matière de vaccination que d'environnement (ondes électromagnétiques par exemple).

25. Entretien avec l'auteure, 13 juillet 2016.

26. Dans le cadre d'une réforme européenne de la pharmacovigilance, l'enregistrement des effets indésirables dans la base de données européennes est réalisé directement par les industriels titulaires des «Autorisations de mises sur le marché», depuis 2014. Pour son analyse de la pharmacovigilance, dont l'organisation est pilotée en France par l'Agence nationale de sécurité du médicament et des produits de santé, le rapport mobilise les critiques formulées par une revue professionnelle médicale qui revendique son indépendance à l'égard des laboratoires pharmaceutiques : la revue Prescrire est ainsi citée à la p. 39 du rapport et son directeur fait partie des personnes auditionnées, au titre de «personnalité qualifiée». 
auteurs « soulignent que les membres (de l'organe consultatif national) doivent déclarer d'éventuels conflits d'intérêt, doivent se sentir libres et encouragés à exprimer leurs points de vue même s'ils sont en contradiction avec celles des gestionnaires du programme de vaccination ou les politiques du ministère de la santé [...] Les modes de fonctionnement décrits comme optimaux sont assez proches de ceux du CTV et notamment l'absence de participation de l'industrie aux réunions» (Hurel, 2016, p.53).

Le rapport convoque donc une diversité d'acteurs - institutionnels, scientifiques et professionnels mais aussi non-institutionnels, certains de ces groupes étant en situation de conflit ouvert dans d'autres arènes - associations versus industries, par exemple, ou de relative ignorance - associations et acteurs de la santé publique notamment - et étant de fait très rarement en situation de concertation et encore moins de dialogue.

Même si leur légitimité à intervenir dans la redéfinition de la politique vaccinale n'est pas affirmée comme étant équivalente, le rapport établit ce faisant un état des lieux relativement atypique en ce qu'il est profondément conflictuel, lequel avait jusqu'alors été rendu impossible, pour ne pas dire interdit, par les acteurs institutionnels, politiques et scientifiques légitimes considérant communément que «la vaccination, ça ne se discute pas » 27 .

Cette position dogmatique fait l'objet d'un constat, établi par les deux premières phrases du rapport :

La politique vaccinale a fait l'objet de controverses et ce depuis de très nombreuses années. Le rôle majeur de la vaccination en santé publique, avec son succès tangible lié à des baisses de mortalité et de morbidité pour des maladies graves, avait conduit la plupart des scientifiques et les pouvoirs publics à écarter pendant longtemps ce sujet du débat. (Hurel, 2016, p.3)

Les associations de patients, tout comme les firmes pharmaceutiques, se trouvent ainsi conduites à exposer plus publiquement leurs positions, ce qui peut les amener sinon à diversifier leurs logiques d'action, du moins à devoir se positionner par rapport à des logiques d'actions diversifiées, du fait des contraintes propres à tout débat public et de la nécessité de se conformer à des règles de publicisation et de débat équivalentes - règles auxquelles les uns et les autres échappent tant qu'ils évoluent de manière privilégiée et séparée dans les réseaux sociaux numériques ou dans les espaces discrets de l'action publique.

27. Citation de la ministre de la Santé, Marisol Touraine, en réponse à des questions de journalistes sur le succès de la pétition initiée par H. Joyeux, le 29 mai 2015. La déclaration fait alors l'objet de deux dépêches de l'Agence France-Presse et devient une véritable «petite phrase » compte tenu de sa reprise et de sa circulation dans de très nombreux médias pendant plus d'un mois. 


\section{La publicisation de difficultés et de débats internes aux institutions du système de santé : le rapport comme vecteur de déconfinement}

Le rapport est par ailleurs significatif de tensions ou de dysfonctionnements qui se déploient dans les institutions et les agences sanitaires, et aussi entre elles - autrement dit dans ces «espaces au sein desquels se négocient les orientations de l'action publique» sur la base de compromis ou de «transactions collusives » entre des acteurs aux logiques et aux intérêts différents » (Gilbert et Henry, 2012).

Ressortent en premier lieu et de manière saillante «la complexité du système » et la nécessité - exprimée de manière fortement prescriptive - de clarifier et d'expliciter les prérogatives et les missions des différentes instances, ainsi que de réinstaurer un pilotage reconnu.

L'adhésion à la politique vaccinale nécessite une transparence et une clarté des messages qui suppose un pilotage du système où chacun des acteurs de la politique vaccinale trouve sa place. (Hurel, 2016, p. 51) ${ }^{28}$

Cette partie du rapport dresse un tableau dysfonctionnel des institutions et des agences de santé publique responsables de l'élaboration de la politique vaccinale et tend à se départir de la neutralité souvent observée dans ce genre de production institutionnelle. Les pages consacrées à rappeler les attributions des institutions sanitaires, leurs relations hiérarchiques et les missions des agences sanitaires indépendantes signifient en effet leur morcellement, leur insuffisante coordination, la complexité des circuits de circulation des informations ainsi qu'une carence de pilotage tant sur le plan hiérarchique que fonctionnel.

Les missions, le positionnement de la DGS et l'acuité des sujets liés à la vaccination doivent la conduire à renforcer ce pilotage. (Hurel, 2016, p. 51)

Recommandation n' 5 : Les échanges entre l'ANSM, I'ONIAM (Office national d'indemnisation des accidents médicaux) et la DGS devraient être davantage formalisés afin de potentialiser les connaissances en matière de survenue et d'indemnisation des effets indésirables. (Ibid., p.52)

Le CTV effectue donc un travail de fond permettant la mise à jour des recommandations et l'élaboration de nouvelles recommandations [...] Les liens étroits entre ANSM, CTV et InVS [Institut national de veille sanitaire, devenu l'Agence nationale de santé publique au 1er mai 2016] conditionnent la réactivité de la qualité des analyses. (Ibid.)

28. Le titre de la sous-partie 3.3 du chapitre 3 est intitulé «L'adhésion à la vaccination ne peut pas être considérée comme acquise d'emblée». 
Enfin, l'insuffisance des liens entre CTV et HAS, bien que la HAS soit membre du CTV, ne permet pas de renforcer la cohérence entre les décisions des différentes commissions [...] Un rapport de l'IGAS [Inspection générale des affaires sociales] de janvier 2015 préconisait également le rattachement du CTV à la HAS. Il est également indispensable que les membres de l'InVS, de l'INPES et de l'ANSM participent, sans voix délibérative comme c'est le cas actuellement, aux réunions de cette nouvelle entité. (Hurel, 2016, p.54-55)

La dimension prescriptive du rapport repose donc en grande partie sur ce constat d'une gouvernance lacunaire. Elle est encore manifeste dans le «plan d'action pour la rénovation de la politique vaccinale » annoncé par la ministre de la santé à l'occasion de la remise du rapport, et dont le deuxième axe s'intitule «Coordonner : assurer une meilleure gouvernance de la politique vaccinale » 29 .

Des tensions internes au système constitué par les institutions et les agences sanitaires apparaissent de manière saillante, sur deux sujets en particulier.

Le premier est l'objet principal du rapport, à savoir la pertinence de maintenir la distinction entre des vaccins obligatoires et des vaccins recommandés. La redéfinition de ces notions, initialement prévue dans le «programme national d'amélioration de la politique vaccinale 2012-2017", a fait l'objet d'avis divergents dans les arènes confinées de l'action publique. Les résultats d'une enquête réalisée en 2007 par l'Institut national de veille sanitaire et l'Institut national de promotion et d'éducation pour la santé sur la perception de l'obligation vaccinale par la population et par les médecins (Gautier, 2008) font craindre que sa disparition induise une chute de la couverture vaccinale des vaccins obligatoires et des vaccins recommandés combinés aux vaccins obligatoires. La Commission des Affaires sociales de l'Assemblée nationale (France, 2012) ainsi que l'Académie nationale de pharmacie (2012) se sont du coup prononcées pour un élargissement de l'obligation au vaccin rougeole-oreillons-rubéole. En revanche, la Conférence nationale de santé, organe consultatif créé en 1996 auprès du ministère, s'est déclarée comme n'étant «pas opposée » à la substitution de l'obligation «par une notion de recommandation associée à un niveau de priorité et de pertinence appuyé sur des preuves scientifiques périodiquement évaluées, prenant en compte les enjeux de santé publique » (Conférence nationale de santé, 2012). Enfin tout en restant également très pondéré, l'avis du Haut Conseil de la santé publique de mars 2013 et 2014 considère que le «maintien d'une obligation vaccinale » constitue seulement une «hypothèse » dont la validation devrait être accompagnée d'une révision de la liste des vaccins obligatoires (Haut Conseil de la santé publique, 2014, p.5). Pour ce qui est des arènes médiatiques, accessibles au grand public, le quotidien La Croix signale par ailleurs dans un article en date du 15 septembre 2015, faisant état du débat public sur l’obligation vaccinale

29. Communiqué de presse du ministère des Affaires sociales, de la Santé et des Droits des femmes, 12 janvier 2016. 
annoncé par la ministre à l'issue de la remise du Rapport Hurel, que «certains de ses membres estiment qu'il faut supprimer le caractère obligatoire de la vaccination DT-polio [...] Ces experts jugent qu'il faut en finir avec la distinction entre les vaccins “obligatoires" et ceux seulement "recommandés" comme le ROR (Rougeole-oreillons-rubéole). Cette distinction finit, selon eux, par créer la confusion dans une partie du public qui juge que les vaccins recommandés sont moins importants ou moins utiles que ceux qui sont obligatoires».

Tout en faisant porter sa première recommandation sur l'impossibilité d'en rester à un «statu quo dans ce domaine», le rapport sur la politique vaccinale fait donc très explicitement état des divergences discrètes - au sens où elles sont peu publicisées - entre agences sanitaires et sociétés savantes, lesquelles impliquent le recours à un débat public par ailleurs soutenu par d'anciens responsables de la santé publique ${ }^{30}$.

Le second sujet significatif de tensions internes au système de santé est la modification des lieux et des acteurs de la vaccination. L'analyse du rapport fait ressortir l'ambivalence des syndicats de médecins de ville, entre conservatisme corporatiste et manque d'implication d'une part, et revendication de leurs compétences et de leur «licence» quasiment exclusive (Hughes, 1996) de procéder à la vaccination d'autre part - puisque « $85 \%$ des vaccinations se font dans le système libéral en France ». Même si le Rapport Hurel réaffirme la «place essentielle et incontournable des médecins de ville dans le dispositif de vaccination» (Hurel, 2016, p.72), il évoque plusieurs exemples de pays dans lesquels les pharmaciens procèdent à la vaccination et souligne que «les infirmiers jouent également un rôle majeur dans la vaccination dans d'autres pays» (Hurel, 2016, p.73). Cette fois encore, le conflit interne au système de santé français est décelable grâce aux corpus complémentaires, qui viennent éclairer l'insistance sur la comparaison internationale ainsi que cette mention allusive, présente dans le texte du rapport :

Sans débattre ici de son rôle comme effecteur de la vaccination (cf. comparaisons internationales), le pharmacien apparaît comme un acteur essentiel compte tenu de ses obligations générales de conseil, d'orientation vers un praticien qualifié et de pharmacovigilance. (Hurel, 2016, p.65)

Le Conseil national de l'Ordre des pharmaciens, qui fait partie des organisations rencontrées pour l'élaboration du rapport, rappelle régulièrement

30. Comme par exemple l'ancien directeur général de la santé William Dab, qui écrit sur son blog, en pleine controverse médiatique sur la pétition Joyeux et suite à la déclaration de la ministre selon laquelle «la vaccination ça ne se discute pas» : «Une stratégie d'intervention fondée sur l'obligation doit se discuter non dans ses principes, mais dans ses résultats, et ceux-ci ne sont pas si favorables que l'on puisse se dispenser d'un débat dont la Conférence nationale de santé aurait pu être saisie». W. Dab, «Vaccins obligatoires : rassurer, inquiéter ou éclairer? À propos de la pétition Joyeux», Des risques et des hommes : incertitudes et démocratie, 〈http://securitesanitaire.blog.lemonde.fr/2015/06/07/vaccins-obligatoires-rassurer-inquieter-ou-eclairera-propos-de-la-petition-joyeux/> (consulté le 5 avril 2017). 
l'exemplarité des nombreux pays qui autorisent la vaccination par les pharmaciens d'officine. Dans un communiqué de presse en date du 12 juillet 2016 faisant suite à l'annonce de l'ouverture de la concertation citoyenne sur la vaccination, il se déclare «favorable à ce que, dans un avenir proche, le pharmacien d'officine puisse vacciner les adultes, sous certaines conditions (dont la transmission de l'information de vaccination aux médecins), et pour certains vaccins, comme c'est déjà le cas dans de nombreux pays) ».

Enfin l'information et surtout la formation des médecins par les institutions sanitaires et la Direction générale de la santé en particulier apparaissent comme des enjeux majeurs - elles font l'objet de trois recommandations sur vingt. L'implication des praticiens de ville, dont les syndicats représentatifs ont été entendus pour élaborer le rapport, apparaît douteuse et sujette à caution. En témoignent plusieurs passages du rapport évoquant le temps insuffisant que les médecins consacrent à la mise à jour de leurs connaissances comme à l'information des patients sur la vaccination, ainsi que les commentaires de la députée lorsqu'elle explique que les syndicats en font un argument de la revalorisation du prix de la consultation ${ }^{31}$.

Lors des auditions, les médecins ont plusieurs fois insisté sur le temps passé à expliquer l'intérêt des vaccinations qui va en augmentant dans le contexte actuel. (Hurel, 2016, p. 6)

Le site de l'ANSM constitue une source précieuse d'informations mais tous les acteurs, et en particulier les médecins prescripteurs, n'ont pas forcément le temps de le consulter. (Ibid., p.49)

Leur comportement [des médecins généralistes] en termes de suivi des recommandations dépendra notamment de leur confiance dans les autorités et de leur perception de l'utilité et les risques des vaccins. (Ibid., p. 44)

Le déconfinement opéré par le rapport se situe également sur le plan procédural, par la recommandation de la création d'un «comité des parties prenantes »:

Le comité, présidé par le DGS, comporterait les différentes parties prenantes : des professionnels de santé, des usagers, des représentants d'associations impliqués dans la maîtrise des risques liés à la vaccination ou à la non-vaccination, des scientifiques [...] Il sera sollicité régulièrement pour faciliter la compréhension mutuelle des différents acteurs et éclairer la décision publique en matière de vaccination. (Hurel, 2016, p. 52 : «Recommandation $n^{\circ} 7 »$ )

Dans l'organisation du rapport, cette recommandation précède l'ultime et importante recommandation relative à l'organisation d'un débat public sur l'obligation vaccinale : tout se passe comme si elle transposait au fonctionnement des arcanes de santé publique les principes qui ont présidé à son élaboration.

31. Entretien avec l'auteure le 13 juillet 2016. 
A bien des égards, le processus de déconfinement dont le rapport est le vecteur apparaît donc conforter l'hypothèse selon laquelle le problème public de la vaccination est en partie lié à des tensions internes - et pas uniquement à des mises en cause extérieures et plus ou moins profanes : «l'instruction des problèmes, leur mise en forme s'opèrent donc habituellement au sein de groupes de spécialistes, dans des espaces recevant une faible attention publique, les débordements dans l'espace public étant souvent (bien que pas exclusivement) la marque de ruptures de compromis, d'effritements des alliances réalisées autour de la définition d'un problème» (Gilbert et Henry, 2012, p. 51).

\section{Conclusion}

De prime abord, le « rapport sur la politique vaccinale» respecte globalement les attendus de ce genre de production institutionnelle. Commandé à une élue ayant eu à mettre en œuvre une campagne de vaccination dans sa circonscription, il vise à instruire un projet de transformation de la politique publique française émanant initialement d'une institution sanitaire, sur la base d'un état des lieux et d'auditions et en proposant vingt « recommandations» qui sont bien mises en valeur dans la composition du texte et lui confèrent une dimension fortement prescriptive.

Ce rapport n'en constitue pas moins un espace discursif inédit, en ce qu'il rend compte d'une polyphonie jusqu'alors inexistante, puisque de fait morcelée en plusieurs espaces "d'interactions et d'interlocutions» (Cefaï, 1996, p. 47) fondés sur l'asymétrie de l'accès aux différentes arènes publiques et médiatiques et sur le confinement d'une partie des débats entre experts et acteurs institutionnels.

Si le texte rappelle de manière récurrente et insistante les bénéfices sanitaires et médicaux de la vaccination, fondateurs de la politique publique, c'est en opérant néanmoins des reformulations significatives de l'attention prêtée aux contre-discours qui contestent les autorités institutionnelle et scientifique hégémoniques, lesquels contre-discours sont le plus souvent récusés par les autorités politique et scientifique - puisque « la vaccination, ça ne se discute pas». Lorsque le rapport souligne, à plusieurs reprises, la nécessité d'informer très largement sur les «risques liés à la non-vaccination» ou encore les « risques évités par le vaccin ${ }^{32}$, c'est en contrepoint de la reconnaissance des «risques individuels de certains vaccins», position constitutive des associations de patients mais aussi, avant elles, des mouvements anti-vaccination. Cette mise en balance des principes et des arguments fondateurs de la poli-

32. Hurel, 2016, p. 57 : «3.3.6. Des échanges réguliers doivent être organisés avec les usagers et les associations impliquées dans la maîtrise des risques liés à la vaccination ou à la nonvaccination». 
tique publique de la vaccination, opérée dans le rapport, en fait le lieu d'une polyphonie élargie, qui fait une place inédite à des discours antérieurement tenus à l'écart, et qui se trouve ainsi traversé par des rapports de force entre des groupes d'acteurs diversifiés : institutionnels, scientifiques et professionnels et non-institutionnels - firmes pharmaceutiques et associations de patients.

Ce rapport constitue également une étape et un instrument dans un processus de redéfinition de la politique vaccinale, l'une de ses préconisations majeures portant sur l'organisation d'une démarche de concertation - de fait mise en œuvre entre juin et novembre 2016. À ce stade, on ne saurait déterminer quelles sont ses chances de succès. On peut en revanche souligner le caractère agonistique de l'arène singulière constituée par la concertation en question (Mouffe, 2004), laquelle réunit des acteurs et des voix dont les divergences et les conflits apparaissent pour le moment très difficilement dépassables.

Comment garantir le principe de réciprocité, selon lequel les interlocuteurs emploient des arguments qu'ils savent pouvoir être acceptés par l'autre (Blondiaux, 2008), par exemple entre les firmes pharmaceutiques et les associations de patients? Comment faire entrer dans un régime de discussion et de négociation des groupes dont l'autorité - scientifique et professionnelle - est pour l'essentiel fondée sur des résultats considérés comme scientifiquement rationnels? De manière plus générale, une concertation est-elle possible dans un contexte où les intérêts et les logiques d'action diffèrent de manière radicale et où la création d'un «espace commun d'échange agonistique avec leur(s) adversaire(s)» (Rennes, 2016) est sans précédent? Au contraire, peut-on «concevoir des dispositifs de délibération qui ne chercheraient pas à abolir les conflits, mais au contraire parviendraient à en favoriser l'expression » (Blondiaux, 2008)?

Autrement dit, et pour en revenir à l'objet du présent article, le « rapport sur la politique vaccinale», espace polyphonique inédit, constituera-t-il dans l'histoire des rapports de santé publique le prélude à une arène agonistique proprement dite, ou une ouverture éphémère?

\section{Références}

AKRICH Madeleine, RABeharISOA Volona, 2012, "L'expertise profane dans les associations de patients, un outil de démocratie sanitaire», Santé publique, vol.24, p. 69-74.

Bertrand Anne, Torny Didier, 2004, Libertés individuelles et santé collective. Une étude socio-historique de l'obligation vaccinale. Rapport final, Villejuif, Centre de recherche médecine, sciences, santé et société (CNRS UMR 8559, INSERM U502, EHESS) 〈https://halshs.archives-ouvertes.fr/halshs-00397364〉 (consulté le 24 mars 2017). 
BLONDIAUX Loïc, 2008, «Démocratie délibérative vs. démocratie agonistique ? Le statut du conflit dans les théories et les pratiques de participation contemporaines », Raisons politiques, vol.2, n³0, p.131-147.

CEFAï Daniel, 1996, «La construction des problèmes publics. Définitions de situations dans des arènes publiques ", Réseaux, nº 75, p. 43-66.

CEFAï Daniel, 2009, «La fabrique des problèmes publics. Boire ou conduire il faut choisir!», postface à J. Gusfield, La culture des problèmes publics, Paris, Economica, p. 219-318.

Cefaï Daniel, TRom Danny, 2005, «Retour sur la sociologie des problèmes publics. Un entretien avec Joseph Gusfield », Secret/Public, 2005, p. 209-222.

GAUTIER Arnaud éd., 2008, Enquête Nicolle 2006: connaissances, attitudes et comportements face au risque infectieux, Saint-Denis, Éd. de l'INPES.

GILBERT Claude, Henry Emmanuel, 2012, «La définition des problèmes publics : entre publicité et discrétion », Revue française de sociologie, nº 1, vol. 53, p. 35-59.

Grant Lenny, Hausman Bernice L, CAShion Margaret, Lucchesı Nicholas, Patel Kelsey, RoBERTS Jonathan, 2015, «Vaccination Persuasion Online: A Qualitative Study of Two Provaccine and Two Vaccine-Skeptical Websites», Journal of Medical Internet Research, vol.17, p.1-20.

GUSFIELD Joseph, 2009 [1981], La culture des problèmes publics, Paris, Economica.

Hilgartner Stephen, Bosk Charles L., 1988, «The Rise and Fall of Social Problems: A Public Arenas Model», American Journal of Sociology, vol. 84, p. 53-78.

HUGHES Everett, 1996, Le regard sociologique, Paris, Éd. de l'EHESS.

Juhem Philippe et SEDEL Julie éd., 2016, Agir par la parole. Porte-paroles et asymétries de l'espace public, Rennes, PUR.

Moulin Anne-Marie, 1996, L'aventure de la vaccination, Paris, Fayard.

Mouffe Chantal, 2004, "Le politique et la dynamique des passions», Rue Descartes, n० $45-46$, p. 179-192.

NEVEu Erik, 1999, «L'approche constructiviste des “problèmes publics”. Un aperçu des travaux anglo-saxons », Études de communication, n² 22, p. 41-58.

OLLIVIER-YANIV Caroline, 2016, «La vaccination, ça se discute? Analyse de la trajectoire médiatique d'une controverse des réseaux sociaux aux médias légitimes », 6es Journées d'étude du «Pôle Santé \& Société » de l'Université Paris-Est: Agents infectieux et vaccins chez l'homme et l'animal, École nationale vétérinaire d'Alfort: 30-31 mars 2016, 〈http://journee-sante-societe.fr/fr/retour-sur-les-6es-journees-d-etude/ document-2701.html> (consulté le 24 mars 2017).

RENNES Juliette, 2016, "Les controverses politiques et leurs frontières », Études de communication, $\mathrm{n}^{\circ} 47, \mathrm{p} .21-48$.

TORNY Didier, 2011, «La politique vaccinale en situation controversée durable», communication au Congrès pluri-thématique de la Société française de Santé publique: les expertises en santé publique. Lille : 2-4 novembre 2011, 〈http://www.canal-u. tv/video/canal_u_medecine/sfsp_lille_2011_la_politique_vaccinale_en_situation_controversee_durable.7640> (consulté le 24 mars 2017).

VILDÉ Jean-Louis, 2015, «L’obligation vaccinale en question », Laennec, t. 63, nº 3, p. 8-23. 
WARD Jeremy, 2015, Les vaccins, les médias et la population : une sociologie de la communication et de la représentation des risques, Thèse de doctorat : Sociologie, Université Paris-Diderot.

Ward Jeremy K., Peretti-Watel Patrick, Larson Heidi, Raude Jocelyn, Verger Pierre, 2015, "Vaccine-Criticism on the Internet: New Insights Based on French-Speaking Websites», Vaccine, n³3, p. 1063-1070.

\section{Documents}

ACAdÉmie NATIONALE DE PHARMACIE, 2012, La vaccination : un acte individuel pour un bénéfice collectif, Recommandations adoptées par le Conseil du 24 octobre 2012, suite à séance thématique du 17 octobre 2012, disponible sur Internet : 〈http://www.acadpharm.org/dos_public/Recommandations_SEance_vaccination_17_10_2012_VF_du_24.10_2012_Conseil.pdf〉 (consulté le 5 avril 2017).

CONFÉRENCE NATIONALE DE SANTÉ, 2012, Avis du 21 juin 2012 portant sur le programme d'amélioration de la politique vaccinale 2012-2017, 〈http://social-sante.gouv.fr/ IMG/pdf/avispolvaccinale2106ninoav030712-2.pdf〉 (consulté le 5 avril 2017).

France, Assemblée nationale, Commission des Affaires sociales, 2012, Rapport d'information $\mathrm{n}^{\circ} 4334$ sur la prévention sanitaire, J.-L. Préel, 8 février 2012.

France, SÉnAt, Commission des AffaiRes sociales, 2013, Rapport d'information sur l'étude de la Cour des comptes relative à la politique vaccinale de la France, G. Labazée, 13 février 2013.

HAUt CONSEIL DE LA SANTÉ PUBLIQUE, 2014 [2013], Avis relatif à la politique vaccinale et à l'obligation vaccinale en population générale (hors milieu professionnel et règlement sanitaire international) et à la levée des obstacles financiers à la vaccination, 13 mars 2013 et 6 mars 2014, 〈http://www.hcsp.fr/Explore.cgi/Telecharger?NomFic hier=hcspa20140306_poletobligvaccinalepopgene.pdf> (consulté le 5 avril 2017).

HUREL Sandrine, 2016, Rapport sur la politique vaccinale [remis au Premier ministre], janvier 2016; disponible sur Internet : 〈http://social-sante.gouv.fr/IMG/pdf/rapport_sur_la_politique_vaccinale_janvier_2016_.pdf〉 (consulté le 5 avril 2017). 